\title{
Research on the Index System of Coordination Effectiveness for Equipment System-of-System based on Information Measurement
}

\author{
Zheng Wang \\ College of Information System and Management, National University of Defense Technology, Changsha, Hunan \\ joshuakai900901@163.com
}

\begin{abstract}
Through physical domain, information domain and cognitive domain are involved in the analysis of coordination effectiveness evaluation for equipment SOS, the index design method based on information measurement is used to design the index system of coordination effectiveness for equipment SOS.
\end{abstract}

\section{Introduction}

Research on coordination effectiveness evaluation method of equipment SOS is an important part of demonstration of equipment SOS, while optimal selection of index system is a key to coordination effectiveness evaluation of equipment SOS [4]. Generally, information measurement is defined as evaluation and measurement on the amount of information. Theory on information measurement provides new technical support for the research on the index system of coordination effectiveness evaluation for equipment SOS. On the basis of difficulties and characteristics of the index system selection of equipment SOS effectiveness, the index system of coordination effectiveness evaluation for equipment SOS based on information measurement is established [5].

\section{Principles Development on the Index System of Coordination Effectiveness Evaluation For Equipment $F$}

Evaluation index is a basic principle to measure combat effectiveness of equipment SOS, has an important directive, normative and tractive effort on military struggle preparation and army construction. When developing the evaluation index, if many problems cannot be solved effectively, such as more subjective qualitative evaluation indexes, fewer objective quantitative evaluation indexes, more single evaluation tests, less system matched evaluation, much static single inspection examination, and less dynamic overall inspection, inspection and evaluation are difficult to truly reflect combat capability of forces. Under conditions of informationization, when developing the combat effectiveness evaluation index, high efficiency and accuracy are required as follows:

\subsection{Evaluation Index Must Be Consistent with Actual Combat Requirement}

Actual combat is the basic standard to examine the combat capability of forces. Inspection and evaluation of combat effectiveness for equipment SOS should objectively response to the combat capability of forces, and develop the evaluation index of coordinative combat effectiveness for equipment SOS in accordance with actual combat standards.

\subsection{Evaluation Index Must be Reasonable and Scientific.}

Coordinative combat of equipment SOS is a complex system integrated comprehensively by various capabilities. Each capability contains several specific capabilities. Therefore, when determining evaluation index, the level should be divided reasonably at first. According to each specific capability required to perform combat mission under conditions of informationization, decomposition is made around combat element, combat unit, combat system, combat SOS and combat direction, key link and key element are chosen to reflex each capability to the greatest extent, weight values of quantification are established scientifically, and quantification evaluation of coordinative combat effectiveness is achieved.

\subsection{Evaluation Index Must be Comprehensive and Measurable.}

Related factors involved in coordinative combat effectiveness of equipment SOS have many types and high correlation. Firstly, when developing the evaluation index, it must be relatively complete and systematic, both 
comprehensively reflex the combat level of equipment SOS and take care of important factors to influence the combat effectiveness of system. Secondly, you should try to select the evaluation index that can go through direct measurement, be obtained by statistics, and be easy to quantification processing, so the influence of human factor is reduced and objectivity, authenticity and operability of evaluation is improved.

\subsection{Evaluation Results Should be Comparable.}

Whether the evaluation index of coordinative combat effectiveness for equipment SOS is reasonable, concerning whether to scientifically measure the gap between combat capability and wining standard by evaluation, compare the combat capabilities under different conditions, evaluate capabilities required to execute different missions by the same force. Therefore, the evaluation index framework needs to be widely recognized and normative. On this basis, detailed index system is developed in accordance with equipment type, mission type, etc.

\section{Evaluation Index System Framework of Coordination Effectiveness For Equipment SOS}

Evaluation index of equipment's combat effectiveness can be progressively analyzed layer-by-layer in four aspects, from bottom to top:

Capability measurement of equipment sub-system;

Single effectiveness measurement: relationship is built with capability index, capability index with same or similar attribute is integrated as a single effectiveness index;

System effectiveness measurement: under certain combat environment, evaluation is made on system effectiveness of key links at every combat stage;

SOS (system-of-system) effectiveness measurement: effectiveness index is determined by comprehensively considering mission and task of equipment SOS, combat scenario, combat unit and other factors between system effectiveness and SOS effectiveness [6].

Layered architectural diagram of combat effectiveness evaluation index system is shown as Figure 1.

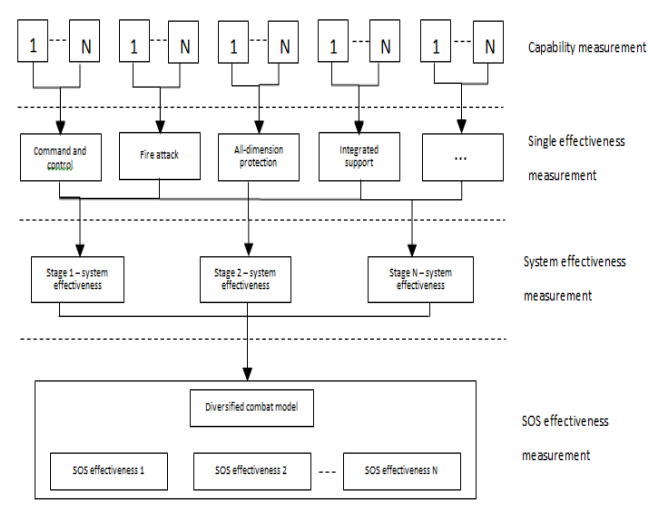

Figure1. Layered architectural diagram of combat effectiveness evaluation index system

Combat effectiveness index measures the size of combat effectiveness, and reflects the implementation extension of combat mission. Selection of effectiveness index affects the validity of combat effectiveness evaluation analysis. When making combat effectiveness evaluation analysis, it is required to reasonably choose combat effectiveness index, sort out relationship between indexes, and establish combat effectiveness index system. Currently, there isn't a set of unified combat effectiveness index to establish rules. Different combat effectiveness indexes can be used by different researchers under different combat scenarios. For example, indexes are presented in terms of deployment maneuver capability, command and control capability, strike and assault capability, defense and survival capability, sustainable combat capability; indexes are presented at the aspect of command information, fire application, battlefield maneuver, comprehensive protection, equipment support, man-machine integration. Some researches on comprehensive evaluation index of combat effectiveness for equipment SOS mainly investigate the following aspects and establish corresponding indexes:

\subsection{Evaluation Index of Intelligence Reconnaissance Capability}

Intelligence reconnaissance is defined as an activity to obtain Intelligence required by military struggle. Evaluation of intelligence reconnaissance capability should make comprehensive survey around whether quantity and types of early-warning and reconnaissance equipment are within a complete range, whether configuration of early-warning and reconnaissance equipment is reasonable, whether detection range can be connected effectively, whether reliability and timeliness of various intelligence obtained by early-warning and reconnaissance forces meet requirements, whether intelligence required by superior leaders can be provided in time, and whether anti-jamming capability of various earlywarning and reconnaissance equipments and their working performance and operator proficiency under various complex 
meteorology and hydrology conditions and complicated electromagnetic environment satisfy requirements, etc.

\subsection{Evaluation Index of Command and Control Capability}

Command and control is a key factor to decide whether the combat is won or not. Its evaluation index should be determined from three main aspects - control coordination structure, control coordination approach and autonomous coordination capability:

Control coordination structure mainly depends on perfection degree of structure establishment, definition degree of duties segregation, smoothness degree of control coordination mechanism, and improvement degree of control coordination efficiency.

Control coordination approach can primarily be measured in two aspects. One is hardware facilities, the other is software facilities. The index of hardware facilities contains the complete extent of information network used to control coordination, the number of distributed integrated information processing center, interconnection extent between each sensor unit and data processing center, networked level between each distributed information processing center, the average number of high-performance military computer used for information processing, average networked level of computers in information processing center, digitized degree of each information carrier, average data storage capacity of each information processing center, etc. The index of software facilities contains the type of application software system for special information processing, the grade of application software system for special information processing, the number of special basic combat database, the application degree of advanced information processing technology, etc.

Autonomous coordination capability is mainly the efficiency of autonomous coordination mechanism between each service force on the basis of mandatory control coordination [7].

\subsection{Evaluation Index of Fire Strike Capability}

This index development mainly considers the following aspects: able to give full play to the performance of weapon and equipment; able to apply tactics flexibly based on the change of battlefield situation and complete combat mission in accordance with specified time; suppressive fire, air defense fire, anti-tank fire, anti-ship fire, anti-submarine fire, to-space fire, to-air fire, to-earth fire, and to-sea fire can exert each advantage to strike against enemy accurately and effectively; whether the probability of damage meets specified requirements, etc.

\subsubsection{Evaluation Index of Stereoscopic Maneuver Capability}

It consists of air maneuver capability, sea maneuver capability, ground maneuver capability. Evaluation of stereoscopic maneuver capability should develop evaluation index in terms of scramble, rapid maneuver, engagement preparation, adaptability at random, etc. Evaluation index of scramble contains whether to be able to achieve combat readiness transfer within specified time, whether real time of scramble can meet requirements, and whether sortie rate of staff and weapon equipment can meet standards; Evaluation index of rapid maneuver contains whether matching transportation scheme is sophisticated and reasonable, all kinds of situation disposal in the march is effective in time, whether safe protection measures are efficient, and whether staff and equipment van reach specified location and forceful situation is established against enemy; Evaluation index of engagement preparation contains whether force assemble and deployment can meet tactical requirements, whether to perform combat marshalling and organize combat mobilization in accordance with combat requirements, and whether everything is ready for operation within specified time; Evaluation index of adaptability at random contains whether quick response can be made at any time in accordance with the change of battlefield situation, whether disposal is timely and effective, whether redeployment is rapid and concealed, etc.

\subsubsection{Evaluation Index of Information Attack Capability}

Evaluation index can be divided into quantity, quality and applied level of offensive equipments in various information war. Specific index contains quantity and performance of ground fixed electronic interference station in the battlespace, quantity and performance of aircraft in electronic warfare into which combat controls can be put, quantity and performance of anti-radiation UAV, proportion of satellite communications jamming equipment, quantity and performance of equipment in tactical electronic warfare, average quantity of ship-based electronic jamming equipment, quantity and performance of ship-based acoustic jamming equipment, means of network attack against enemy, able to identify jamming characteristics of enemy timely and accurately, and probability of destroying communication system of enemy, etc.

\subsubsection{Evaluation Index of All-Dimension Protection Capability}

It should cover defensive reconaissance and surveillance, air defense anti-missile, information defense and other protective action. Evaluation index of air defense anti-missile mainly contains whether various air defense forces allocations are reasonable, and can form long-range, midrange and short-range, high-altitude, mid-altitude and low- 
altitude air defense anti-missile system, early-warning detection distance, range, probability and time of air strike forces against enemy and incoming missile, response time of air defense anti-missile troops, resist capability of air strike forces against enemy and incoming missile, etc. Index of information defense capability mainly contains physical protective capability, technology protective capability, and safe management capability index [8].

\subsubsection{Evaluation Index of Integrated Support Capability}

Evaluation mainly contains three aspects - combat support, logistics support and equipment support. Evaluation index of combat support, logistics support and equipment support should mainly assess whether support force is grouped uniformly and used scientifically, whether support task allocation is reasonable, whether support relationship is clear, whether support plan is sophisticated, whether various support material, weapon equipment, ammunition and storage can achieve specified index, whether support method is applied flexibly, whether support is real-time, timely and effective, etc.

\subsubsection{Evaluation Index of "Three-War" Capability}

It mainly contains whether advocacy is achieved timely and effectively to make friendly forces maintain morale high; whether to go to psychological warfare widely in order to break down the morale of enemy effectively; whether various media and information resources can be used effectively to make friendly forces maintain advantageous position in public opinion and to make hostile forces passive position in public opinion; whether to make full use of international law, law of war and other favorable conditions to disclose various illegal behaviors and ways of enemy in the war; whether legal basis is chosen to be good for friendly forces to go to war in existing international law, law of war and other international regulations, etc.

\subsection{Index System of Coordination Effectiveness for Equipment SOS based on Information Measurement}

\subsubsection{Information Conversion in Equipment SOS.}

Logically, structure of equipment SOS contains three domains: Physical Domain, Information Domain and Cognitive Domain, as shown in Figure 2. Physical Domain mainly contains combat force of both sides, various combat platforms and geographic space, etc. Information Domain contains various sensors used to detect information about enemy, fusion devices used to deal with information, and communication network used to transfer information [1]. Cognitive Domain contains command and control device used to make decision by commander and assistant commander. Main work performed in three domains is as follows:

Physical Domain is used to describe battlefield facts, including objective physical object - weapon system, topographic features, troops, etc. Physical Domain also contains intangible content, such as combat intention and combat plan of both sides [3].

Information Domain deals with data from Physical Domain, form and distribute Common Relevant Operating Picture (CROP). Three main functions are achieved: collection of data from sensor; generation of CROP by fusion processing; distribution of CROP to all friendly forces.

Cognitive Domain is the cognitive process of CROP. Individuals receive CROP and generate situation awareness of individuals, and form shared situation awareness and make decision by teamwork. Its workflow is: the objective battlefield information from Physical Domain is used to detect, integrate, comprehensively deal with, transfer and generate $\mathrm{CROP}$ in Information Domain; commander cognizes CROP, forms situation awareness, makes decision in accordance with battlefield situation, and commands and controls troops.

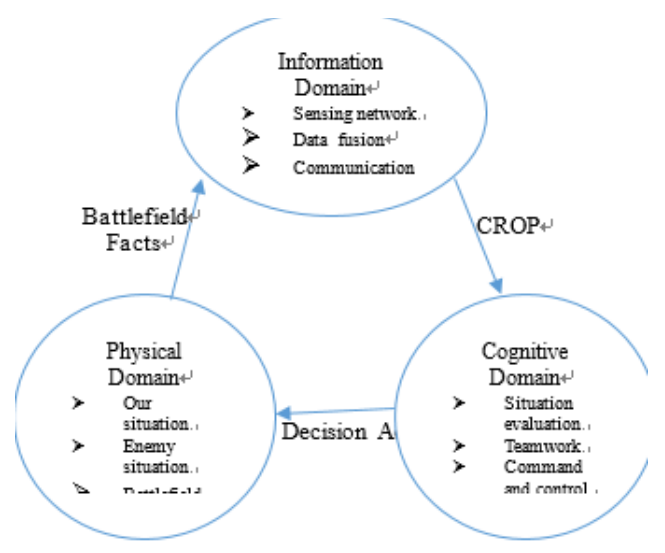

Figure.2. System structure diagram of equipment SOS

Coordination combat of system is mainly defined that all sub-systems coordinate with each other by electronic information system, the key point of which is quality of information transferred between electronic information systems, mainly including six steps:

Battlefield facts reception: weapon battlefield facts are received from Physical Domain;

Data acquisition: sensor network is used, battlefield space entity is detected, and related battlefield facts data is acquired;

Data fusion: at the first stage, data is transferred to the fusion device to deal with various intelligence; at the second stage, fused data of each fusion system is transferred to central processing device to generate CROP;

Network distribution: according to demands of troops, different versions of CROP are transferred to user by network; 
Individual awareness: each user recognizes and understands received CROP to get certain battlefield situation awareness. CROP is explained alone, whose quality is affected by technology and capability of individuals;

Shared awareness: battlefield situation facts are perceived between individuals by coordination improvement, finally shared situation awareness is formed [2].

Above processes are linked to obtain the information conversion link of information system, as shown in the figure. Hierarchical conversion is described in Figure 3, whose emphasis is placed on quality of processes instead of processes themselves. Two in Information Domain, while three in Cognitive Domain, described as follows:

The first conversion describes the impact of sensor capability on quality of information acquired by sensor. The change in information quality of sensor described in figure can be gained by changing comprehensive degree and characteristic parameters of sensor network.

The second conversion represents quality of information from sensor group. Quality of CROP is formed after fusion. Fusion index refers to information fusion level of battlefield entity by fusion device.

During the third conversion, CROP generated by fusion is distributed to user to observe CROP quality via network. Observable CROP quality depends on communication network of information transmission. Different versions of CROP are distributed to different users, which depends on demands of end-users.

The fourth conversion is that the information is transferred to Cognitive Domain, the relationship between observable CROP and individual awareness is described, and the quality of individual awareness depends on capability level of individuals.

The fifth conversion describes the relationship between individual situation awareness and shared situation awareness. Situation awareness serves as dependent variable to replace information quality. This is the beginning of teamwork. Coordination capability relies on several factors, such as team experience, teamwork time, personality of members, etc.

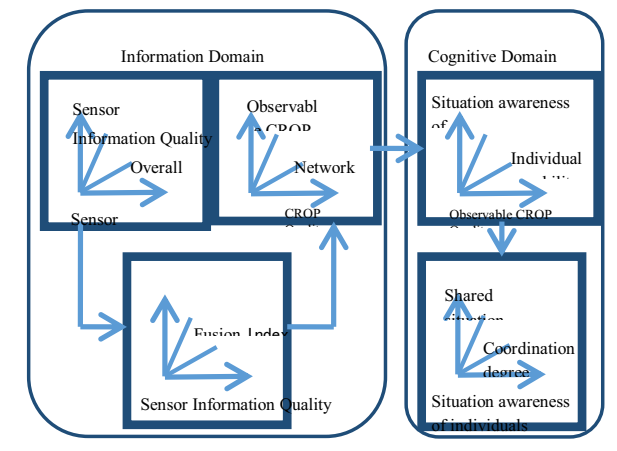

Figure.3. Links diagram of system information conversion

\subsubsection{Evaluation Index System Based On Information Superiority}

Above all, information superiority of system is measured by analyzing the quality of each information conversion process in equipment SOS, to evaluate the coordinative combat effectiveness of equipment SOS. Information superiority reflects contrast results of information countermeasure capability of two sides in the information countermeasure process of information system, finally it is reflected in the quality of shared situation awareness generated in information system, so the quality of available information quantitatively describes information superiority. Due to different forms of information conversion in Information Domain and Cognitive Domain, the quality of information conversion mainly relies on objective factors in Information Domain, such as information acquisition capability, information distribution capability, information defense capability, etc. However, the quality of information conversion mainly relies on subjective factors in Cognitive Domain, such as information processing capability, information sharing degree, decision-making time, etc. Therefore, evaluation indexes of information superiority measurement are different in these two domains. Specific indexes are shown in Figure 4, as indicated below:

Information superiority index

It contains information acquisition capability, information distribution capability, information processing capability and information defense capability. Information acquisition capability is defined as the capability to obtain information from Physical Domain based on the capability of sensor. Information distribution capability is defined as the capability to transfer information in Information Domain based on the quality of network connections. Information processing capability is defined as the capability to do information fusion in Information Domain based on fusion index. Information defense capability is defined as the antijamming capability of information conversion device in Information Domain. commanding and decision-making capability index

It contains the quality of situation awareness, the degree of information sharing, the index of decision-making time, etc. The quality of situation awareness is defined as the quality of shared situation awareness after individual awareness is summarized in accordance with the degree of coordination, one of conclusive indexes based on information superiority. The degree of information sharing is defined as the coordinative degree in Cognitive Domain. The index of decision-making time is defined as absolute time of commanding and decision-making. combat capability index

It contains battle damage, combat achievement, etc. Battle damage is defined as quantity and types of our battle damage units in a simulation countermine experiment. Combat achievement is defined as quantity and types of enemy's battle damage units in a simulation countermine experiment. 


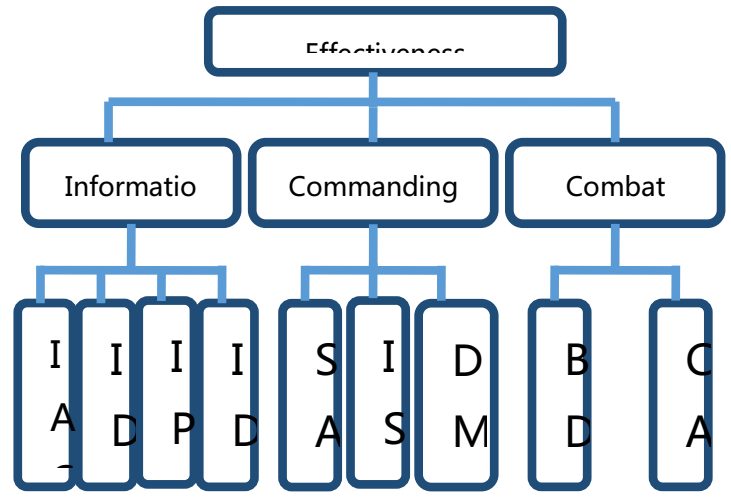

Figure .4. Index system of effectiveness evaluation based information superiority

Notes:IAC- Information acquisition capability; IDC- Information distribution capability; IPC- Information processing capability; IDC - Information defense capability; SAQ- Situation awareness quality; ISD- Information sharing degree; DMT- Decision-making time; BD - Battle damage; CA - Combat achievement

\section{Case}

Above evaluation index system based on information superiority designs a set of simple air combat simulation experiment. Its combat scenario is that: blue side is equipped with a piece of fighter in combat, while red side has the following two schemes.

Table1. Formation scheme of red side

\begin{tabular}{|c|c|c|}
\hline Type & Early warning aircraft (pcs) & Fighter (pcs) \\
\hline $\begin{array}{c}\text { Scheme } \\
1\end{array}$ & 1 & 3 \\
\hline $\begin{array}{c}\text { Scheme } \\
2\end{array}$ & 0 & 4 \\
\hline
\end{tabular}

Evaluation index system based on information superiority is used to analyze the index of combat effectiveness of red and blue sides in above schemes. According to suggestions of domain expert, information acquisition, processing, distribution, and defense capabilities, the quality of situation awareness, and the degree of information sharing are taken value in $[0,1]$; decision-making time is taken value in $(0,1)$, the shorter the decision-making time is, the higher the value is; combat achievement and battle damage are taken value in $[0,1]$. In addition, when performances of fighters of red and blue sides are close, evaluation is made on all basic capability indexes of red and blue sides, as follows.
Table2. Combat effectiveness of red and blue sides

\begin{tabular}{|c|c|c|c|}
\hline Side & $\begin{array}{c}\text { Red Side } \\
\text { Scheme1 }\end{array}$ & $\begin{array}{c}\text { Red Side } \\
\text { Scheme2 }\end{array}$ & Blue Side \\
\hline $\begin{array}{c}\text { Information acquisition } \\
\text { capability }\end{array}$ & 0.6 & 0.2 & 0.2 \\
\hline $\begin{array}{c}\text { Information processing } \\
\text { capability }\end{array}$ & 0.5 & 0.3 & 0.2 \\
\hline $\begin{array}{c}\text { Information } \\
\text { distribution capability }\end{array}$ & 0.5 & 0.2 & 0.2 \\
\hline $\begin{array}{c}\text { Information defense } \\
\text { capability }\end{array}$ & 0.5 & 0.2 & 0.2 \\
\hline $\begin{array}{c}\text { Situation awareness } \\
\text { quality }\end{array}$ & 0.7 & 0.3 & 0.4 \\
\hline $\begin{array}{c}\text { Information sharing } \\
\text { degree }\end{array}$ & 0.7 & 0.8 & 1 \\
\hline Decision-making time & 0.5 & 0.5 & 0.8 \\
\hline Combat achievement & 0.7 & 0.9 & 0.5 \\
\hline Battle damage & 0.7 & 0.6 & 0.7 \\
\hline Overall effectiveness & 0.009 & 0.00016 & 0.00018 \\
\hline
\end{tabular}

It can be seen that each single effectiveness of red side is superior to that of blue side in scheme 1 ; in scheme 2 , only combat capability of red side is superior to that of blue side, and others are close to those of blue side, but its situation awareness quality and decision-making time are lower than those of blue side due to the needs of coordination. Calculation of single effectiveness and SOS effectiveness can use method of weighting, multiplicative method, additive method, etc. In this case, multiplicative method is used, i.e. each single effectiveness is multiplied to get system effectiveness, each system effectiveness is multiplied to get SOS effectiveness(certainly, different evaluation methods of SOS effectiveness can be chosen in accordance with different needs of system evaluation.). In case 1 , case 2 and case 3 , computed SOS combat effectiveness of red side is $0.009,0.00016$ and 0.00018 respectively. This shows that in computed coordinative effectiveness evaluation based on information measurement, the effectiveness of one fighter is higher than that of multiple fighters.

The above discussion can be verified in the following Xsim simulation. As shown in Figure 5, 6, 7, 8, under certain conditions, if red side encounters with blue side in accordance with scheme 1 , before blue side discovers red side's warplane, red side's early warning aircraft have discovered blue side's warplane, finally blue side's warplane is shot down under the coordination between early warning aircraft and fighter and our warplane is not damaged.

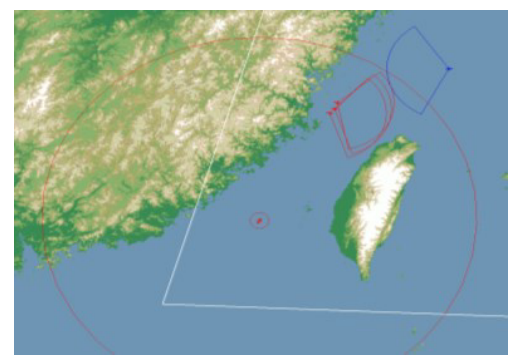

Figure.5. Scheme 1-intial battlefield 


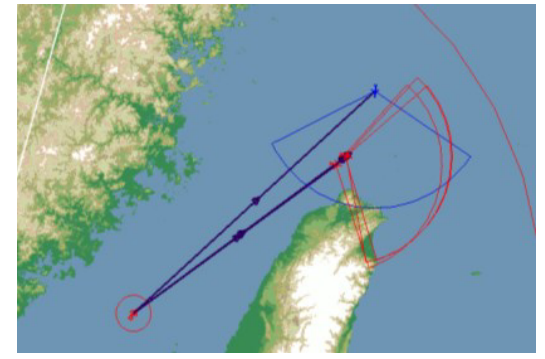

Figure.6. Scheme 1-enemy aircraft detected by early warning aircraft

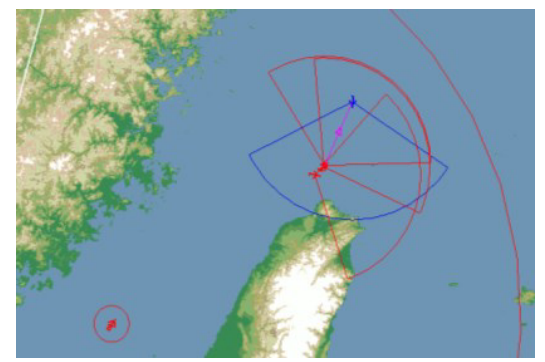

Figure.7. Scheme 1- coordinative operation between early warning aircraft and fighter

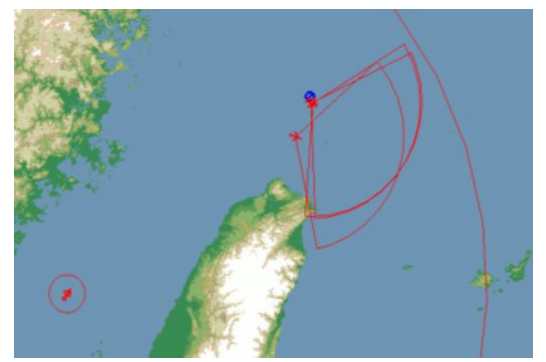

Figure.8. Scheme 1-Blue warplanes are shot down

Under the same conditions, if the formation of red side is based on scheme 2, due to the heading, red side passes blue side and doesn't discover blue side; however, blue side has detected red side and comes round to the back of red side for surprise attack; finally, blue side's warplane is shot down, as shown in figure 9-14.

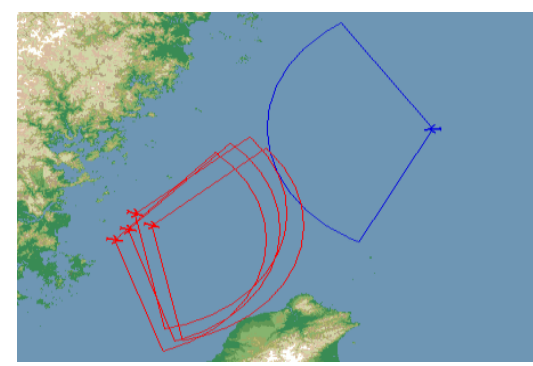

Figure.9. Scheme 2-initial battlefield

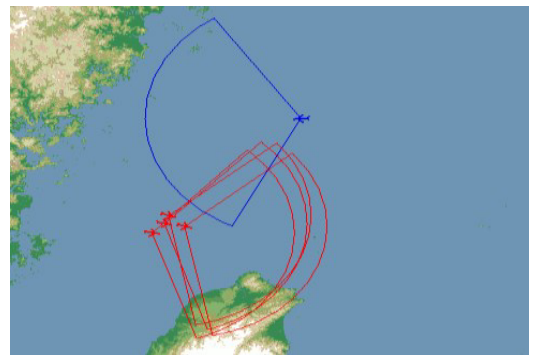

Figure.10. Scheme 2- red side encounters with blue side

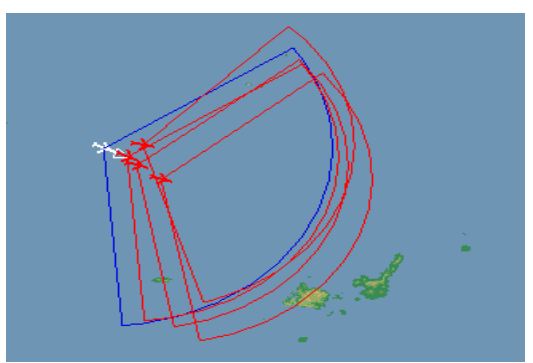

Figure. 11. Scheme 2-surprise attack of blue side from the rear

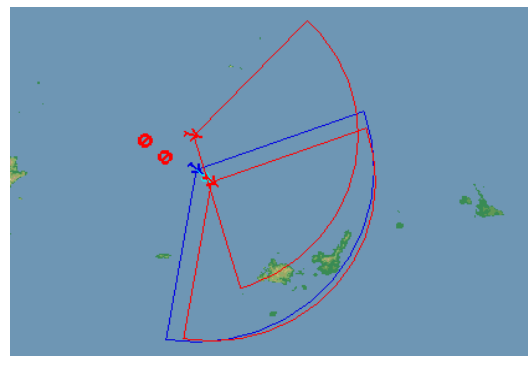

Figure.12. Scheme 2-two red aircraft are shot down

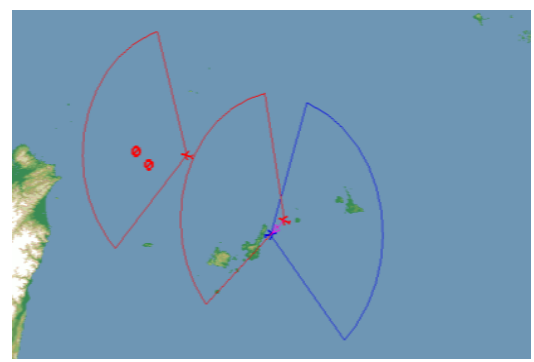

Figure.13. Scheme 2-meeting engagement of red side and blue side(the first warplane is not shot )

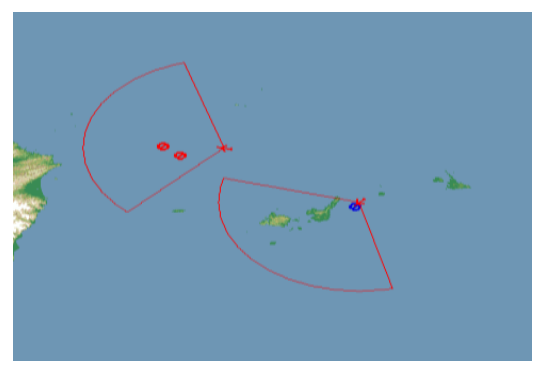

Figure.14. Scheme 2- meeting engagement of red side and blue side(blue side is shot down) 
Above simulation results verify the formation mode of red side in scheme 1, which has better operational effects. Therefore, the index of coordinative effectiveness for equipment SOS based on information measurement has a certain practical significance for the simulation of equipment SOS.

\section{Follow-On Work}

Discussion is made on the selection principles of the index of coordinative effectiveness for equipment SOS. On this basis, it proposes and verifies a index system of coordinative effectiveness for equipment SOS based on information measurement. At the aspect of specific index classification for above index system, it also needs further standardization studies. Meanwhile, conversion rules between different-level indexes also need to be improved further.

\section{References}

[1] Zhi Qunceng, Zhang Letian, Li Zhendong. Information measurement model research based on the state of time dimension [J]. National Defense Science \& Technology, 2015(01):35-39. (references)

[2] Xu Jianfeng, Tang Jun, Ma Xuefeng et al. Objective information model and measurement [J]. SCIENCE CHINA Information Sciences, 2015(03):336-353.

[3] Wang Huanchen. System information measure [J]. Journal of University of Shanghai for Science and Technology, 2011(06):631-640.

[4] Liu Xing, Zhao Min. The measurement of information and knowledge $[\mathrm{J}]$. Command Information System and Technology, 2013(05):39-45.

[5] Walter Perry, Robert W Button, Jerome Bracken et al. Measures of Effectiveness for the information-age navy : The Effects of Network-centric Operations on Combat Outcomes [R]. RAND, 2002.

[6] Tong Huan, Wang Jianzhong, Duo Yinxian, Combat Effectiveness Evaluation of Firearms system based on MMESE, International Conference on Information, Network and Automation, 2010.

[7] Zheng Xuebing, heng Jinjin, The fuzzy Synthetic Evaluation of Combat Effectiveness for Avionics System based on Rough set Theory,International Forum on Information Technology and Applications,2009

[8] Jerry Wightman, The Role of Modeling and Simulation in the Evaluation of FCS: OneSAF, NETS, and DETES, 2008.4.9. 